\title{
Study of Error Performance of Rotated PSK modulation in Nakagami-q (Hoyt) Fading Channel
}

\author{
Kapil Gupta \\ Department of Electronics \& \\ Communication Engineering, \\ Faculty of Engineering and \\ Technology, Mody Institute of \\ Technology and Science \\ (Deemed University) \\ Lakshmangarh, Dist. Sikar \\ Rajasthan (INDIA)
}

\author{
P.K.Ghosh \\ Department of Electronics \& \\ Communication Engineering, \\ Faculty of Engineering and \\ Technology, Mody Institute of \\ Technology and Science \\ (Deemed University) \\ Lakshmangarh, Dist. Sikar \\ Rajasthan (INDIA)
}

\begin{abstract}
The paper concerns with the study of the modulation diversity in PSK scheme due to rotation of the signal constellation along with bit interleaving. We derive here the expressions for the Symbol Error Probability (SEP) of rotated BPSK and QPSK modulations over frequency non-selective slowly fading Nakagami-q channels. Numerical results based on moment generating function (MGF) are obtained and comparison of error performance are made for conventional and rotated modulation schemes. We investigate also the dependence of symbol errors on signal-to-noise ratio, Nakagami-q factor and the angle of rotation. The results show significant improvement in the performance of rotated scheme over conventional method. The optimum rotation angle is also calculated and a symmetrical nature of SEP with rotation angle is observed.
\end{abstract}

\section{Keywords}

Symbol error rate, Bit interleaving, Diversity, Moment generating function, Nakagami-q fading channel.

\section{INTRODUCTION}

Fading is an important factor in signal degradation and error performance evaluation for all modulation schemes through wireless media. The fading effect can be reduced by applying various diversity techniques on the communication systems [1-4]. We can state diversity as two or more signal paths that fade signals independently. By various diversity techniques such as space, time, code, frequency [5-8], these independent paths can be combined in a way to reduce the signal fading [9]. Last decade has witnessed the researches on modulation diversity obtained by applying a certain rotation to the signal constellation [10-12]. Rotation or modulation diversity incorporates interleaver/de-interleaver pair in the system that process in-phase and quadrature components of the received signals affected differently by independent channel fading coefficients. These fading coefficients called channel state information (CSI) are assumed to be known at the receiver. Of the coherent schemes, PSK modulation is very often preferred for its better spectral efficiency. In this paper, the closed form expressions for error probabilities for rotated PSK on frequency non-selective Nakagami-q channel have been derived. Depending on the environments of the communication system, channel models such as Rayleigh,
Ricean, Nakagami-m, Nakagami-q and many more have been proposed for the statistical description of the amplitude and phase of multipath fading signals. Nakagami [13] introduced Nakagami-q model as an approximation for the Nakagami-m fading distribution in the range of fading that extends from the one sided Gaussian model to the Rayleigh model with the value of $\mathrm{q}$ (Nakagami-q factor) varied from 0 to 1 . This type of distribution was also investigated by Hoyt, and is sometimes referred to as the Nakagami-Hoyt distribution [14]. Recently, this model is being used more frequently to calculate error rate performance and outage analysis in cellular mobile radio communications [15, 16]. One-sided Gaussian fading occurs in the limiting case where the variance of imaginary (or real) part is zero; but, Rayleigh case is obtained when the variances of real and imaginary parts are equal. One-sided Gaussian fading corresponds to the worst case fading [17]. This type of fading is applicable on satellite communication links which are subject to strong ionospheric scintillation [17-19].

The rest of the paper is organized as follows: Section II describes the model of the system using PSK over Nakagami-q fading channels. The Error performance analysis of rotated PSK modulation for both one-sided Gaussian and Rayleigh distribution is presented and discussed in section III. Computed results are presented and discussed in Section IV. Finally, in section V, conclusion is made.

\section{SYSTEM MODEL}

If the conventional PSK signal constellation is rotated by a constant phase angle then it results rotated PSK signal constellation, as shown in Fig. 1.

The square in Fig. 1 represents the signal constellation of rotated PSK by an angle $\phi^{0}$ whereas the small solid circles represent the signal points for conventional PSK modulation. Symbol interleaving does not offer appreciable SNR gain as there is one common component with some other symbols in the constellation of the conventional PSK. But when rotation is applied, the symbols having no common components, the symbol interleaving introduces a diversity gain into a system [20]. 


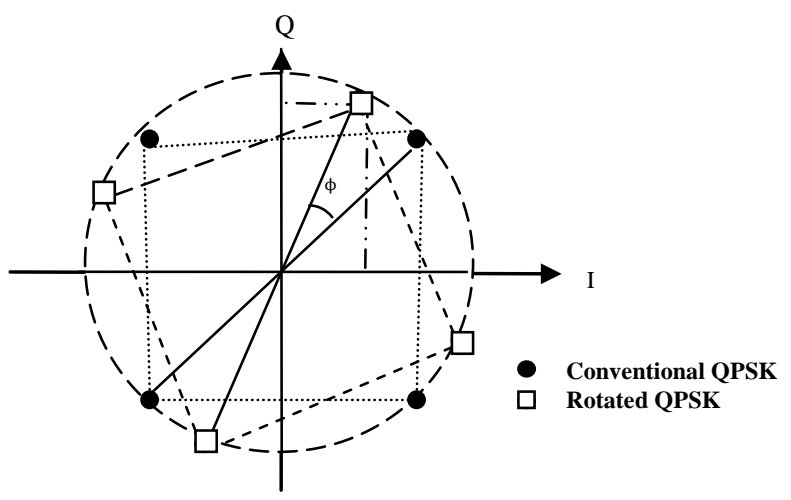

Fig 1: Signal Constellation for Conventional and Rotated QPSK.

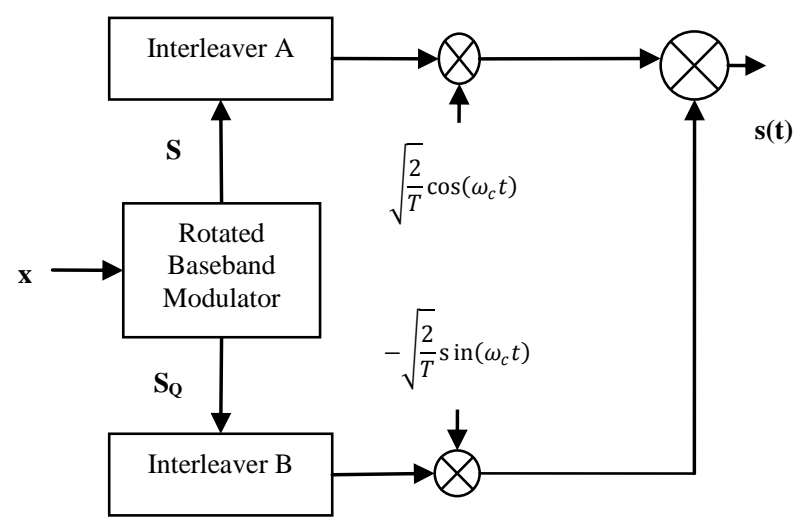

Fig 2: Block diagram of rotated PSK transmitter.

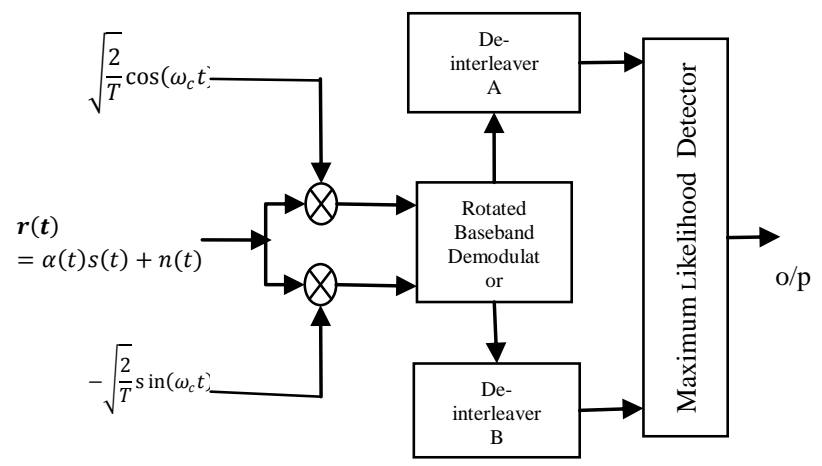

Fig 3: A block diagram representation of rotated PSK receiver.

Rotated modulation scheme should have a different transmitter structure because of the rotation of signal constellation and symbol interleaving as shown in Fig. 2. The rotated PSK modulator necessitates two orthogonal I and Q channels; the in-phase $\left(s_{I}\right)$ and quadrature $\left(s_{Q}\right)$ components of the transmitted signal on two channels are independent from each other. In case of rotating scheme, the bitinterleaved transmitted signal can be written as

$\mathrm{s}(\mathrm{t})=V \sum_{n=-\infty}^{+\infty} x_{n} p(t-n T) \cos \left(\omega_{c} t\right)+V \sum_{-\infty}^{+\infty} y_{n} p(t-$ $n T) \sin \left(\omega_{c} t\right)$

where $x_{n}=a_{n} \cos \phi-b_{n} \sin \phi \quad$, and $y_{n}=a_{n} \sin \phi+$ $b_{n} \cos \phi$ and $V$ represents the modulator gain. The signal is assumed transmitted through additive white Gaussian noise (AWGN) channel.

The interleaver/de-interleaver pair ensures that the in-phase and quadrature components are independent after deinterleaving. The closest constellation points for PSK scheme depends on the rotation angle. The received signal can be written as

$r(t)=\alpha(t) s(t)+n(t)$

where $\alpha(t)$ represents Nakagami-q fading envelope causing random amplitude variation and $\mathrm{n}(\mathrm{t})$ represents zero mean Gaussian noise. In case of slow fading, the fading amplitude is considered constant over one symbol interval. The block diagram for the receiver structure of rotated PSK scheme is shown in Fig. 3. The received signal is applied to the demodulators (two orthogonal carriers $\cos \left(\omega_{c} t\right)$ and $\sin \left(\omega_{c} t\right)$ multiply the received signal in two different branches) and then de-interleaved. The outputs of de-inteleavers are $r_{I}$ and $r_{Q}$, called the in-phase and quadrature components of the received vector $\bar{r}$ and are given by

$\bar{r}=\left(r_{I}, r_{Q}\right)=\left(\alpha_{1} s_{I}+n_{I}, \alpha_{2} s_{Q}+n_{Q}\right)$

For ideal interleavers, $\alpha_{1}$ and $\alpha_{2}$ as independent identically distributed (i.i.d) random variables of zero mean with $E\left[\alpha_{1}^{2}\right]=E\left[\alpha_{2}^{2}\right]=1$. The in-phase and quadrature noise components $n_{I}$ and $n_{Q}$ are also assumed independent random variables with zero mean and variance $\mathrm{N}_{0} / 2$. Since the complete channel state information (CSI) is assumed available at the receiver and the detection is based on maximum likelihood ML decision [21], the detection metric $m\left(\overline{s_{i}} \mid \bar{r}, \bar{\alpha}\right)$ can be obtained as

$m\left(\overline{s_{i}} \mid \bar{r}, \bar{\alpha}\right)=\left\|\bar{r}-\bar{\alpha} \odot \overline{s_{i}}\right\|^{2}, i=1,2, \ldots \ldots, M$.

where $\bar{\alpha}=\left(\alpha_{1}, \alpha_{2}\right)$ and $s_{i}(i=1,2, \ldots . M)$ is the $\mathrm{i}^{\text {th }}$ symbol of rotated PSK constellation, ' $\|$. $\|$ ' denotes the norm and $\odot$ represents component-wise product. The output of ML detection goes in favor of that symbol which minimizes the detection metric.

\section{ERROR RATE ANALYSIS}

\subsection{Rotated PSK modulation}

The conditional bit error probability can be written as [12]

$$
P\left(\bar{s} \rightarrow \hat{s} \mid \alpha_{1}, \alpha_{2}\right)=Q\left(\sqrt{\frac{\alpha_{1}^{2} \Delta_{I}^{2}+\alpha_{2}^{2} \Delta_{Q}^{2}}{2 N_{0}}}\right)
$$


where the vector $\hat{\mathrm{s}}=\left(\hat{\mathrm{s}}_{\mathrm{I}}, \hat{\mathrm{s}}_{\mathrm{Q}}\right)$ denote the in-phase and quadrature components of the estimate of the signal $\bar{s}$, $\Delta_{I}=s_{I}-\hat{s}_{I}, \Delta_{Q}=s_{Q}-\hat{s}_{Q}$, and $\mathrm{Q}($.$) is the Gaussian Q-$ function as [21]. We have Craig's formula of Q-function [22] as

$$
\mathrm{Q}(\mathrm{x})=\frac{1}{\pi} \int_{0}^{\frac{\pi}{2}} \exp \left(\frac{-x^{2}}{2 \sin ^{2} \theta}\right) d \theta
$$

The conditional probability of error event given in Eq. (5), averaged over the probability density function (pdf) of fading amplitude leads to average symbol error rate. By taking $y_{1}=\frac{\alpha_{1}^{2} \Delta_{I}^{2}}{N_{0}}$ and $y_{2}=\frac{\alpha_{2}^{2} \Delta_{Q}^{2}}{N_{o}}$, the average symbol error rate (ASER) can thus be obtained as [17]

$P(\bar{s} \rightarrow \hat{s})=\int_{0}^{\infty} \frac{1}{\pi} \int_{0}^{\pi / 2} \exp \left(\frac{-y}{4 \sin ^{2} \theta}\right) d \theta p_{y}(y) d y$

where $p_{y}(y)$ is the pdf of $y=y_{1}+y_{2}$.

Now, the moment generating function $M_{y}(\epsilon)$ of $y$, defined as

$$
M_{y}(\epsilon)=\int_{0}^{\infty} p_{y}(y) e^{y \epsilon} d y
$$

Thus, equation (7) reads as

$$
P(\bar{s} \rightarrow \hat{s})=\frac{1}{\pi} \int_{0}^{\pi / 2} M_{y}\left(\frac{-1}{4 \sin ^{2} \theta}\right) d \theta
$$

The MGF of Nakagami-q distributed random variable is given by [17]

$M_{y}(\epsilon)=\left(1-2 \in \bar{y}+\frac{(2 \epsilon \bar{y})^{2} q^{2}}{\left(1+q^{2}\right)^{2}}\right)^{-\frac{1}{2}}$

Taking $a=\frac{q^{2}}{\left(1+q^{2}\right)^{2}}$, Eq. (10) becomes

$M_{y}(\epsilon)=\left(1-2 \in \bar{y}+(2 \in \bar{y})^{2} a\right)^{-\frac{1}{2}}$

\subsection{Nakagami-q fading channel}

By Eq. (11), the MGF of $y$ is written as

$$
\begin{aligned}
& \mathrm{M}_{\mathrm{y}}\left(\epsilon=\frac{-1}{4 \sin ^{2} \theta}\right)=\left[1+\frac{2 \bar{y}}{4 \sin ^{2} \theta}+\left(-\frac{2 \bar{y}}{4 \sin ^{2} \theta}\right)^{2} a\right]^{\frac{-1}{2}} \\
& =\frac{4 \sin ^{2} \theta}{\sqrt{\left(4 \sin ^{2} \theta\right)^{2}+2 \bar{y}\left(4 \sin ^{2} \theta\right)+4 a \bar{y}}}
\end{aligned}
$$

Thus, MGF of y can be expressed as $M_{y}(\epsilon)=M_{y_{1}}(\epsilon) \cdot M_{y_{2}}(\epsilon$ ) given in [23]. Then equation (9) becomes

$$
P(\bar{s} \rightarrow \hat{s})=\frac{1}{\pi} \int_{0}^{\pi / 2} M_{y_{1}}\left(\frac{-1}{4 \sin ^{2} \theta}\right) \cdot M_{y_{2}}\left(\frac{-1}{4 \sin ^{2} \theta}\right) d \theta
$$

The conditional error probability is thus obtained as

$$
\begin{aligned}
& P(\bar{s} \rightarrow \hat{s})= \\
& \frac{1}{\pi} \int_{0}^{\frac{\pi}{2}} \frac{4 \sin ^{2} \theta}{\sqrt{\left(4 \sin ^{2} \theta\right)^{2}+2 \overline{y_{1}}\left(4 \sin ^{2} \theta\right)+4 a \overline{y_{1}}}} \frac{4 \sin ^{2} \theta}{\sqrt{\left(4 \sin ^{2} \theta\right)^{2}+2 \overline{y_{2}}\left(4 \sin ^{2} \theta\right)+4 a \overline{y_{2}}}} \mathrm{~d} \theta
\end{aligned}
$$

By substituting $\cos ^{2} \theta=\mathrm{x}$, and $-2 \sin \theta \cos \theta \mathrm{d} \theta=\mathrm{dx}$, the above equation reduces to

$P(\bar{s} \rightarrow \hat{s})=$

$\frac{2}{\pi} \int_{0}^{1} \frac{(1-x)^{\frac{3}{2}} x^{-\frac{1}{2}}}{\sqrt{4(1-x)^{2}+2 \overline{y_{1}}(1-x)+a \overline{y_{1}}} \sqrt{4(1-x)^{2}+2 \overline{y_{2}}(1-x)+a \overline{y_{2}}}} d x$

Case I. If $q=0$ (one sided Gaussian fading) then $a=0$

By putting $a=0$ in Eq. (16), we get

$P(\bar{s} \rightarrow \hat{s})$

$=\frac{2}{\pi} \int_{0}^{1} \frac{(1-x)^{\frac{3}{2}} x^{-\frac{1}{2}}}{\sqrt{4(1-x)^{2}+2 \overline{y_{1}}(1-x)} \sqrt{4(1-x)^{2}+2 \overline{y_{2}}(1-x)}} d x$

$=\frac{2}{\pi} \int_{0}^{1} \frac{(1-x)^{\frac{1}{2}} x^{-\frac{1}{2}}}{\sqrt{4(1-x)+2 \overline{y_{1}}} \sqrt{4(1-x)+2 \overline{y_{2}}}} d x$

$=\frac{1}{2 \pi} \int_{0}^{1} \frac{(1-x)^{\frac{1}{2}} x^{-\frac{1}{2}}}{\sqrt{(1-x)+\frac{\overline{y_{1}}}{2}} \sqrt{(1-x)+\frac{\overline{y_{2}}}{2}}} d x$

By subtituting $b_{1}=1+\frac{\overline{y_{1}}}{2} \quad$ and $\quad b_{2}=1+\frac{\overline{y_{2}}}{2}$ we get

$P(\bar{s} \rightarrow \hat{s})$

$=\frac{1}{2 \pi \sqrt{b_{1}} \sqrt{b_{2}}} \int_{0}^{1}(1-x)^{\frac{1}{2}} x^{-\frac{1}{2}}\left(1-\frac{x}{b_{1}}\right)^{\frac{-1}{2}}\left(1-\frac{x}{b_{2}}\right)^{\frac{-1}{2}}$

The ASER can be expressed in the closed form [24, Eq. 3.211]; which is

$\int_{0}^{1}(1-x)^{\square-1} x^{\lambda-1}(1-u x)^{-\rho}(1-v x)^{-\sigma} d x=$
$B\left([, \lambda) F_{1}(\lambda, \rho, \sigma, \lambda+[? ; u, v)\right.$

where $F_{1}(., \ldots, \ldots, \ldots)$ is the Appell hypergeometric function and $B(a, b)$ is the beta function.

By using the above identity, Eq. (20) reduces to

$$
P(\bar{s} \rightarrow \hat{s})=\frac{B\left(\frac{3}{2}, \frac{1}{2}\right)}{2 \pi \sqrt{b_{1}} \sqrt{b_{2}}} F_{1}\left(\frac{1}{2}, \frac{1}{2}, \frac{1}{2}, 2 ;\left(b_{1}\right)^{-1},\left(b_{2}\right)^{-1}\right)
$$

At high SNR, the hypergeometric fuction converges to one and the upper bound of error rate can be expressed as

$P(\bar{s} \rightarrow \hat{s}) \leq \frac{B\left(\frac{3}{2}, \frac{1}{2}\right)}{2 \pi \sqrt{b_{1}} \sqrt{b_{2}}}$

Case II. For $q=1$ (Rayleigh fading) then $a=0.25$

Eq. (15) becomes:

$P(\bar{s} \rightarrow \hat{s})=$

$\frac{1}{\pi} \int_{0}^{\frac{\pi}{2}} \frac{4 \sin ^{2} \theta}{\sqrt{\left(4 \sin ^{2} \theta\right)^{2}+2 \overline{y_{1}}\left(4 \sin ^{2} \theta\right)+\overline{y_{1}}}} \frac{4 \sin ^{2} \theta}{\sqrt{\left(4 \sin ^{2} \theta\right)^{2}+2 \overline{y_{2}}\left(4 \sin ^{2} \theta\right)+\overline{y_{2}}}} \mathrm{~d} \theta$ 
$=\frac{1}{\pi} \int_{0}^{\frac{\pi}{2}} \frac{4 \sin ^{2} \theta}{\sqrt{\left(4 \sin ^{2} \theta+\overline{y_{1}}\right)^{2}}} \frac{4 \sin ^{2} \theta}{\sqrt{\left(4 \sin ^{2} \theta+\overline{y_{2}}\right)^{2}}} \mathrm{~d} \theta$

$=\frac{1}{\pi} \int_{0}^{\frac{\pi}{2}} \frac{4 \sin ^{2} \theta}{\left(4 \sin ^{2} \theta+\overline{y_{1}}\right)} \frac{4 \sin ^{2} \theta}{\left(4 \sin ^{2} \theta+\overline{y_{2}}\right)} \mathrm{d} \theta$

By substituting $\cos ^{2} \theta=\mathrm{x}$, and $-2 \sin \theta \cos \theta \mathrm{d} \theta=\mathrm{dx}$, and $c_{1}=\frac{\overline{y_{1}}}{4}, c_{2}=\frac{\overline{y_{2}}}{4}$ in Eq. (26), we get

$P(\bar{s} \rightarrow \hat{s})=\frac{1}{2 \pi} \int_{0}^{1} \frac{(1-x)^{\frac{3}{2}} x^{-\frac{1}{2}}}{\left(1-x+c_{1}\right)\left(1-x+c_{2}\right)} d x$

$P(\bar{s} \rightarrow \hat{s})=\frac{1}{2 \pi\left(1+c_{1}\right)\left(1+c_{2}\right)} \int_{0}^{1}(1-x)^{1.5} x^{-.5}(1-u x)^{-1}(1-$

$v x)^{-1} d x$

where $u=\frac{1}{\left(1+c_{1}\right)}$ and $v=\frac{1}{\left(1+c_{2}\right)}$. Since $c_{1}$ and $c_{2}$ are functions of $\phi$, so are $u$ and $v$. Thus the error probability as given in equation (28) depends on $\phi$.

Using Eq. (21) we get

$P(\bar{s} \rightarrow \hat{s})=\frac{B(2.5,0.5)}{2 \pi\left(1+c_{1}\right)\left(1+c_{2}\right)} F_{1}\left[0.5,1,1,3 ;\left(1+c_{1}\right)^{-1},(1+\right.$

$\left.\left.c_{2}\right)^{-1}\right]$.

At high SNR, the hypergeometric fuction converges to one and the upper bound of error rate can be expressed as

$P(\bar{S} \rightarrow \hat{\mathrm{s}}) \leq \frac{B(2.5, .5)}{2 \pi\left(c_{1}\right)\left(c_{2}\right)}$

By assuming that the symbols are of equal probability, union bond for the SEP of M-PSK is expressed as [21]

$P_{S} \leq \frac{1}{M} \sum_{i=1}^{M} \sum_{j=1}^{M} P\left(s_{i} \rightarrow s_{j}\right)$

For binary BPSK case the above equation becomes

$P_{s}=\frac{1}{2}\left[P\left(s_{2} \rightarrow s_{1}\right)+P\left(s_{1} \rightarrow s_{2}\right)\right]$

Similarly for QPSK case we have

$P_{s} \leq \frac{1}{4} \sum_{i=1}^{4} \sum_{j=1}^{4} P\left(s_{i} \rightarrow s_{j}\right)$

The symbol error rate being a function of rotation angle $\phi$, the ASER is required to be minimized with respect to $\phi$ for improved performance. When $\phi=0^{0}$ (without rotation), the PSK signal constellations are pair-wise symmetric with respect to the two axes and the performance of the system reduces to conventional BPSK.

\section{RESULTS}

The study of bit error rate versus the depth of fading is important for assessing the quality of channel. Numerical results are now discussed in this section.

Fig. 4 depicts the variation of SER with the Nakagami-q factor for various signal-to- noise ratio. As $q=0$ corresponds to the worst-case one-sided Gaussian and $q=1$ corresponds to Rayleigh fading, the error performance improves for higher $\mathrm{q}$ values as reflected in Fig. 4. The error rate improves for higher SNR.

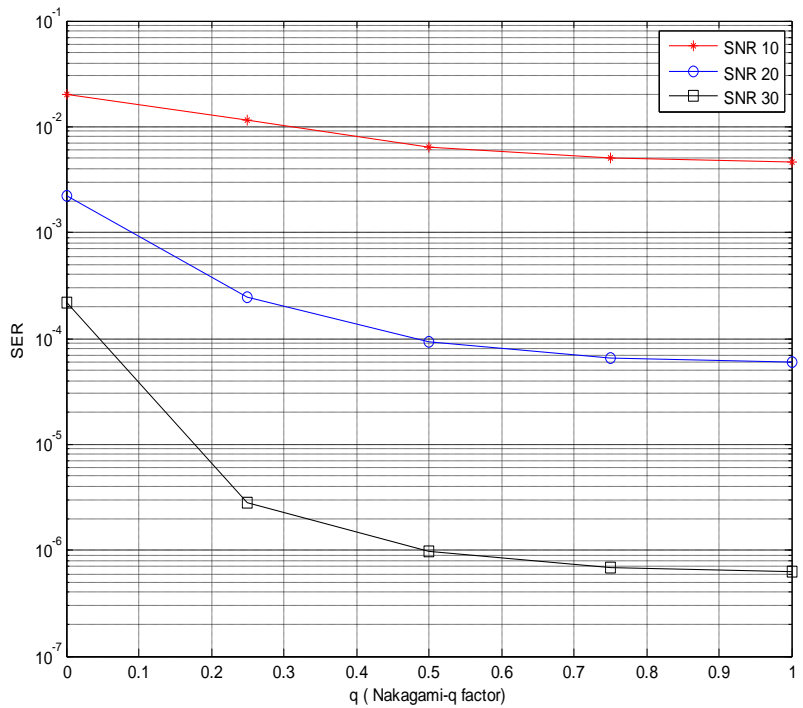

Fig 4: A plot of SER versus Nakagami-q factor for different SNR (dB) values for BPSK at a fixed angle of rotation of $45^{\circ}$.

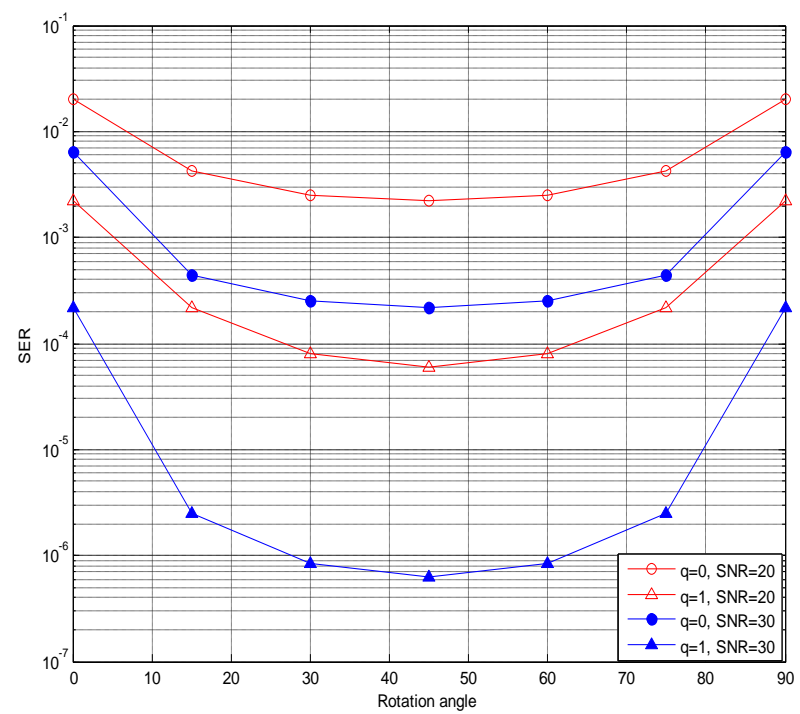

Fig 5: The variation of symbol error rate versus rotation angle for different $\boldsymbol{q}$ and SNR values for BPSK

The SER in Fig. 5 is symmetric with respect to the rotation angle at $45^{\circ}$; this is due the fact that the values of $u$ and $v$ in Eq. (28) are interchanged for the angles $45^{\circ}-\beta$ and $45^{\circ}+\beta$ where $0 \leq \beta \leq 45^{\circ}$. At a given depth of fading (q fixed), the SER is lower for higher SNR values. At a fixed SNR, the performance is improved for higher $\mathrm{q}$. 


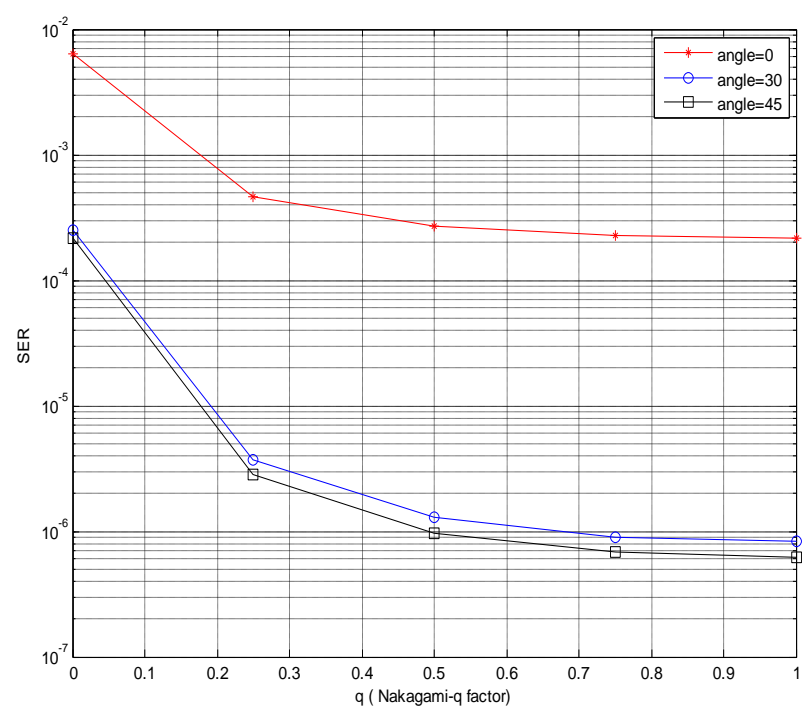

Fig 6: A plot of SER versus Nakagami-q factor for three different rotation angles of $0^{0}, 30^{\circ}$ and $45^{\circ}$ for BPSK at $\mathrm{SNR}=30 \mathrm{~dB}$

Fig. 6 shows that as the rotation angle $\phi^{\circ}$ increases from zero to $45^{\circ}$ through $30^{\circ}$, the SER decreases and attains a minimum at $45^{\circ}$. But with further increase of the rotation angle from $45^{\circ}$ to $90^{\circ}$ there is an increment in SER values. This can be explained in the light of Fig. 5. Also observed here is an improvement in SER as Nakagami-q factor increases in the range 0 to 1 .

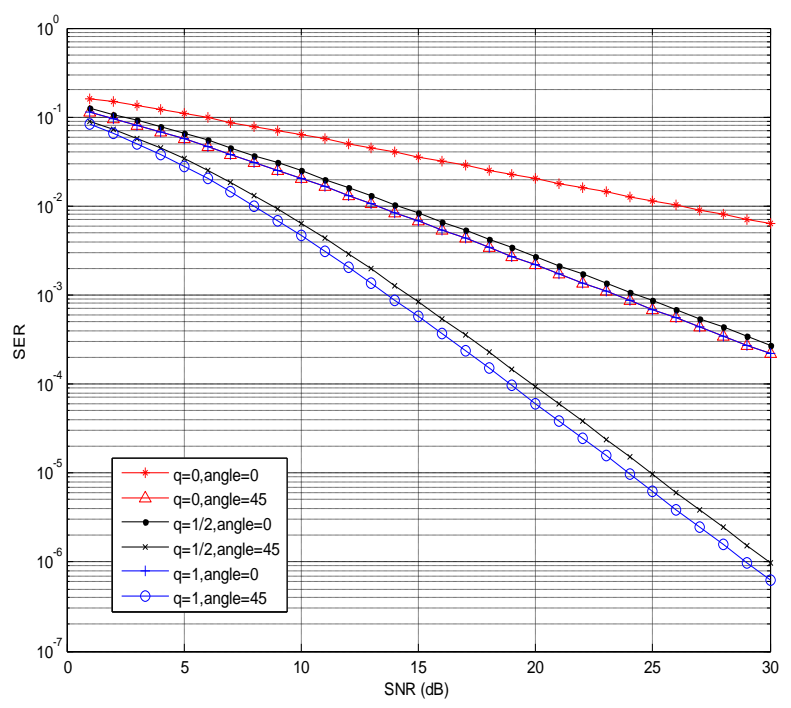

Fig 7: Dependence of SER on SNR various combinations of $q$ and rotation angle.

Similar studies have also been undertaken to find the nature of variation of SER with SNR for different combinations of $q$ and rotation angle, as shown in Fig. 7. Over the range of $q$ from 0 to 1 and the range of rotation angle from $0^{0}$ to $45^{\circ}$, the worst-case combination is found for $q=0, \phi=0^{0}$ and the best-case is for $q=1, \phi=45^{\circ}$.

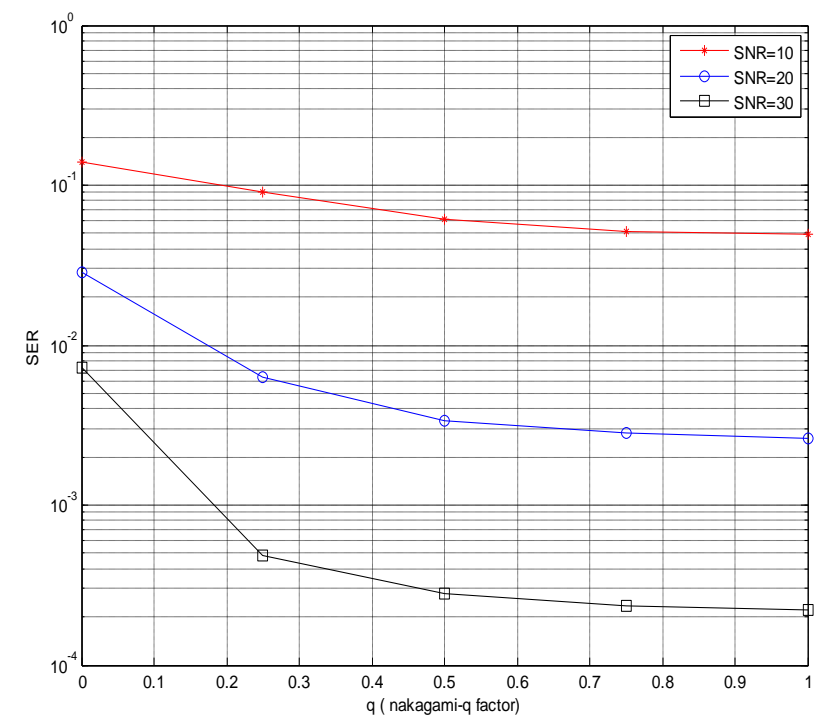

Fig 8: A plot of SER versus Nakagami-q factor for different SNR (dB) values for QPSK at a fixed angle of rotation of $45^{\circ}$.

Because of practical importance, similar studies as above for BPSK have been persuaded for QPSK case also. Fig. 8 shows the variation of SER with Nakagami-q factor for various SNR. We observe here that at a given SNR of $30 \mathrm{~dB}$ and $\mathrm{q}=1$, the SER for QPSK is $2 * 10^{-4}$ against the value of $7 * 10^{-7}$ in BPSK case. The high value of SER in QPSK compared to BPSK for a fixed SNR and $\mathrm{q}$ is due to reduced minimum distance amongst the signal points in the constellation of rotated PSK.

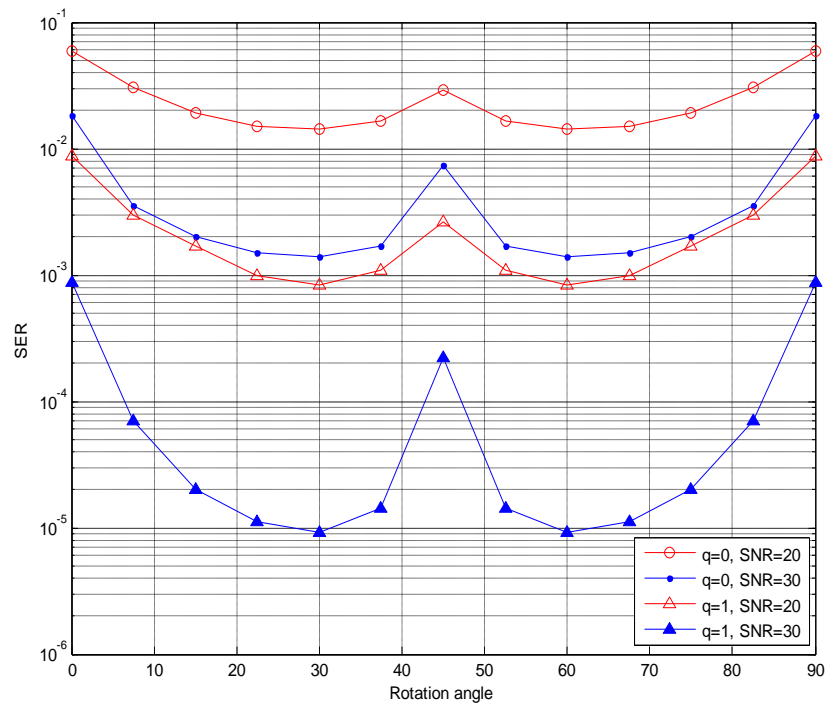

Fig 9: The variation of symbol error rate versus rotation angle for different $\boldsymbol{q}$ and SNR values for QPSK. 
The SER in Fig. 9 is symmetric with respect to the rotation angle at $45^{\circ}$ as explained earlier for BPSK case. At a given SNR, the symbol error rate decreases initially with rotation angle and then increases again with the increase of angle till $45^{\circ}$. This behavior can be explained from the generalized Eq. (22). The term $P\left(s_{i} \rightarrow s_{j}\right)$ contains $1 / \sqrt{b_{1}} \sqrt{b_{2}}$ which is the product $\Delta_{I} \Delta_{Q}$. The dependence of this product on the rotation angle can explain this nature. In our calculation, the optimum angle is obtained close at $30^{\circ}$.

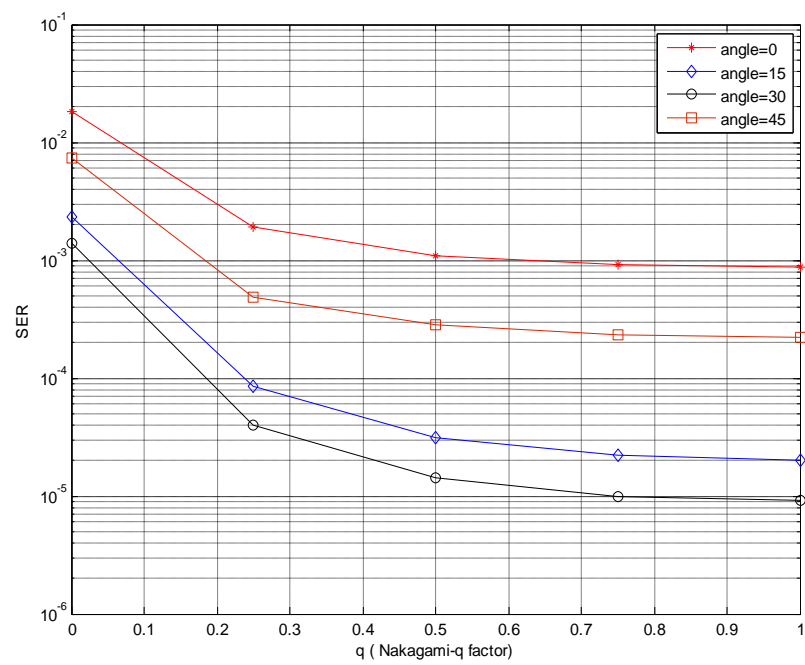

Fig 10: A plot of SER versus Nakagami-q factor for different rotation angles for QPSK at SNR = $30 \mathrm{~dB}$.

Fig. 10 shows that as the rotation angle $\phi$ increases from zero to $45^{\circ}$ through $15^{\circ} \& 30^{\circ}$, the SER decreases with angle till $30^{\circ}$ and attains a minimum value at $30^{\circ}$ (optimum angle) unlike the BPSK case where the minimum value is obtained at $45^{\circ}$.

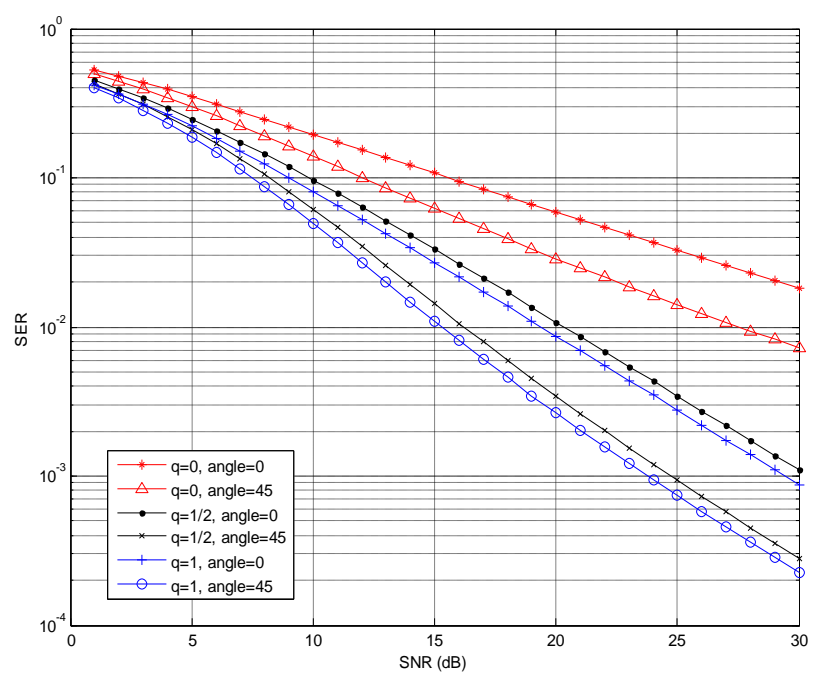

Fig 11: Dependence of SER on SNR various combinations of $q$ and rotation angle for QPSK.
Fig.11 shows the curves between SER \& SNR for rotation angles of $0^{\circ} \& 45^{\circ}$, and $\mathrm{q}=0,0.5$ and 1 . The worst-case combination is found for $q=0, \phi=0^{0}$ whereas the best-case is for $q=1, \phi=45^{0}$, same as the case of BPSK.

\section{CONCLUSION}

In this paper, we have derived the expressions for symbol error analysis using moment generating function for conventional and rotated PSK modulation schemes over Nakagami-q fading channels. We have calculated the dependence of symbol error rates on the modulation techniques (BPSK and QPSK), signal-to-noise ratio, fading parameter $\mathrm{q}$ and the angle of rotation. As the SNR increases, the SER decreases as expected. Our critical analysis shows that the symbol error rates attain minima for the rotation angles of $45^{\circ}$ and $30^{\circ}$ for BPSK and QPSK, respectively. The symmetrical variation of SEP versus rotation angle about $45^{\circ}$ is observed in both the BPSK and QPSK schemes and explained.

\section{REFERENCES}

[1] Jing, Y. and Jafarkhani, H. 2009. "Single and multiple relay selection schemes and their achievable diversity orders", IEEE Trans. on Wireless Communications, , Vol. 8, no. 3, pp. 1414-1423.

[2] Tsiftsis, T. A. 2007. "Performance of Wireless Multihop Communications Systems with Cooperative Diversity over Fading Channels" International Journal of Communications Systems, Wiley, vol. 20, no.12, Dec.

[3] Bletsas, A., Khisti, A., Reed, D.P., and Lippman, A. 2006. "A simple cooperative diversity method based on network path selection," IEEE Journal on Selected areas in Commun., vol. 24, no. 3.

[4] Laneman, J. N., Tse, D. N. C., and Wornell, G. W. 2004. "cooperative diversity in wireless networks: efficient protocols and outage behavior," IEEE Trans. Inform. Theory, Vol. 50, no.12, pp. 3062-3080.

[5] Zhou, Q. and Dai, H. 2007. "Asymptotic analysis on the interaction between spatial diversity and multiuser diversity in wireless networks", IEEE Trans. on Signal Processing, Vol. 55, no. 8, pp. 4271-4283.

[6] Uysal, M. 2006. "Diversity analysis of space-time coding in Cascaded Rayleigh Fading Channels", IEEE Commumn. Let., vol.10, no. 3, pp. 165-167.

[7] Lee, K. and Williams, D. 2000. "A Space-frequency transmitter diversity technique for OFDM systems", Proc. IEEE GLOBECOM, vol. 3, pp. 1473-1477.

[8] Alamouti, S. 1998. "A simple transmit diversity technique for wireless communications", IEEE JSAC, vol. 16,no. 8, pp. 1451-1458.

[9] Andrea Goldsmith. 2005. Wireless Communications, Cambridge University Press.

[10] Sedar $\ddot{Q} z y u r t, ~ O \breve{g u z}$ Kuchur, İbrahim Altunbas, 2007. "Error performance of rotated phase shift keying modulation over fading channels", Wireless Personal Communications. vol. 43, No.04, pp. 1453-1463. 
[11] Slimane, S. B. 1998. "An improved PSK Scheme for fading channels", IEEE Transaction On vehicular Technology, vol 47, no.02, pp. 703-710.

[12] Taricco, G. and Viterbo, E. 1996. "performance of component interleaved signal sets for fading channels", IEE Electronics Letters, vol.32, No.13.

[13] Nakagami, M. 1960. "The m-distribution-A general formula of intensity distribution of rapid fading," in Statistical Methods in Radio Wave Propagation. New York: Pergamon, pp. 3-36.

[14] Hoyt, R. S. 1947. "Probability functions for the modulus and angle of the normal complex variate," Bell Syst. Tech. J., vol. 26, pp. 318-359.

[15] Pornchai, S., Wannaree, W., and Sawasd, T. 2009. "Performance of M-PSK in Mobile Satellite Commuincation over Combined Ionospheric Scintillation and Flat Fading Channels with MRC Diversity," IEEE Transactions on Wireless Communications. Vol. 8(7):3360-3364.

[16] Annamalai, A. C., Tellambura., and Bhargava, V. K. 2001. "Simple and accurate methods for the outage analysis in cellular mobile radio systems-A unified approach," IEEE Trans. Commun., vol. 49, no. 2, pp. 303-316.

[17] Simon, M. K. and Alouini, M. S. 2005. Digital Communication over Fading Channels, 2nd edition, Wiley \& Sons, Inc.
[18] Abdi, A., Lau, W. C., Alouini, M. S., and Kaveh, M. 2003. "A new simple model for land mobile satellite channels: First and second order statistics," IEEE Trans. Wireless Commun., vol. 2, no. 3, pp. 519-528.

[19] Mehrnia, A. and Hashemi, H. 1999. "Mobile satellite propagation channel part II-A new model and its performance," in IEEE Veh. Technol. Conf. (VTC'99), Amsterdam, The Netherlands, pp. 2780-2784.

[20] Yilmaz, A. and Kucur, O., 2009. "Performance analysis of rotated PSK over Nakagami-m fading channels", IEEE 6 th Int. Symp. on Wireless Commun. Systems, Siena, Italy, September pp. 7-10.

[21] Proakis, J. G. 2001. Digital Communications, McGrawHill, New York.

[22] Craig, J. W. 1991. "A new, simple, and exact result for calculating the probability of error for two dimensional signal constellation" in Proc. IEEE milit. commun. conf, MILCOM'91, McLean, VA, pp.571-575.

[23] A. Papoulis. 1991. Probability, Random Variables, and Stochastic Processes, $3^{\text {rd }}$ edition, McGraw-Hill, New York.

[24] Gradshteyn, I. S. and Ryzhik, I. M. 1994. Table of Integrals, Series, and Products, 5th edition, Academic Press, San Diego,

CA. 